SportR Xiv Part of the Society for Transparency, Openness and Replication in Kinesiology (STORK)
Preprint

not peer reviewed

Received: 29 October 2020

Supplementary materials:

For correspondence:

lanburton_10@hotmail.co.uk

\title{
The Effectiveness of Combined Shockwave Therapy and Exercise for Plantar Heel Pain: A Systematic Review Protocol
}

Ian Burton MSc, CSCS.

Physiotherapist, Angus HSCP, NHS Tayside, Dundee, United Kingdom

Please cite as: Burton, I. (2020). The effectiveness of combined shockwave therapy and exercise for plantar heel pain: A systematic review protocol. SportRxiv doi: 10.31236/osf.io/uezfw

\section{ABSTRACT}

Review Objective: To synthesize the best available evidence on the effectiveness of interventions that have used a combination of extracorporeal shockwave therapy and any type of exercise to treat plantar heel pain compared to any other treatment intervention.

Introduction: Recent evidence suggests combining shockwave therapy and exercise may be more effective than other treatments for plantar heel pain. 
However, no systematic reviews have been conducted on the topic and optimal treatment protocols and clinical recommendations are lacking.

Inclusion criteria: Randomised controlled trials assessing the effectiveness of combined shockwave therapy and exercise for plantar heel pain in adults will be included.

Methods: The authors will search for a wide range of sources to find both published and unpublished studies via EBSCOhost, including, but not limited to, MEDLINE, SPORTDiscus, CINAHL, Cochrane Central Register of Controlled Trials (CENTRAL), and Allied and Complementary Medicine Database (AMED). Studies published in a language other than English will only be considered if a translation is available. The JBI systematic review methodology will be followed when conducting the review. Data synthesis will be conducted using meta-analysis or narrative synthesis, where appropriate.

\section{Systematic review registration number: CRD42020213286}

Keywords: Exercise; Muscle Stretching; Extracorporeal Shockwave Therapy: Resistance training; High-Energy Shock Waves; Fasciitis, Plantar.

\section{Introduction}

Recent research on the global burden of disease has highlighted how musculoskeletal disorders are a leading contributor to the global burden of disability and chronic pain [1]. Musculoskeletal disorders have an impact on a broad demographic spectrum of the population, result in extensive expenditure by healthcare systems and are a substantial societal burden [2-4]. However, servicelevel and health policy responses to this burden have been inadequate, with an integrated research and policy agenda required, including prioritising evidencebased effective treatments [5]. Chronic foot pain constitutes a large proportion of the musculoskeletal disease burden, with Plantar Heel Pain (PHP) causing up to $15 \%$ of all foot-related pain complaints and occurring in $10 \%$ of the population [69]. Symptoms of PHP include sharp heel pain commonly felt in the morning or after prolonged sitting, which can cause an impaired gait pattern and functional 
limitations $[10,11]$. The disorder presents like tendinopathy and results in chronic degeneration of plantar fascia tissues and collagen fibres rather than an acute inflammatory response and may result from excessive weight bearing or biomechanical abnormalities $[12,13]$. There are several commonly used clinical treatments for PHP, including exercise therapy, orthotics, corticosteroid injections, non-steroidal anti-inflammatory drugs, taping, manual therapy approaches and platelet rich plasma injections [14-16]. Electrotherapy approaches are also common and include ultrasound, low level laser therapy and extracorporeal shockwave therapy (ESWT) $[17,18]$. Of all currently available treatments, ESWT and exercise approaches appear to have the best evidence of long-term effectiveness [19]. Traditionally, plantar fascia specific stretching has been the most evidence-based exercise approach for PHP [20], however in recent years heavy slow resistance training has been shown to be a more effective form of exercise [21]. Other forms of exercise with inconclusive evidence of effectiveness for PHP include isometric exercise [22], intrinsic foot muscle strengthening [23] and kinetic chain strengthening targeting the hip musculature [24].

After first being successfully implemented in urology as a treatment to disintegrate renal calculi, ESWT is now applied to a range of musculoskeletal disorders such as tendinopathies [25]. For several decades, a significant evidence-base of effectiveness for ESWT has been increasing, particularly for lower limb tendinopathies and PHP [26]. Recalcitrant heel pain that is unresponsive to conservative physiotherapy such as exercise approaches, for three to six months are considered appropriate candidates for ESWT treatment [27]. Several systematic reviews and meta-analyses have concluded there is long-term safety and effectiveness of ESWT compared to placebo or other common PHP treatments, with its use consistently recommended in the literature for PHP [28-32]. In addition, recent evidence suggests that ESWT combined with exercise may be a more effective PHP treatment compared with either of the treatments in isolation [33]. Shockwaves generated by ESWT devices are considered mechanical acoustic energy waves which rapidly create high pressures [34]. This positive high pressure is quickly followed by negative pressure, which returns to ambient values within microseconds [35]. The high pressures generated from shockwaves induce cavitation within human tissue, which involves rapid formation, expansion and 
forceful collapse of vapor bubbles in liquids due to rapid pressure changes [36]. This cavitation process may stimulate a myriad of biological responses that can activate tissue regeneration effects such as stimulating neovascularisation and collagen synthesis in pathological musculoskeletal tissues [37].

In clinical practice, ESWT is rarely used as monotherapy and is more often combined with exercise, with there being a need for a comparison of polytherapy treatment approaches in future studies [38, 39]. Current research recommends that combined rather than isolated treatments be used in PHP treatment, with ESWT or exercise in isolation having inadequate long-term outcomes [13, 40,41]. Despite this increasing evidence for ESWT and exercise in PHP treatment, optimal treatment protocols and clinical recommendations are lacking [42]. There is also a dearth of studies investigating a combined approach with a distinct lack of clinical recommendations on combined protocols, despite indications of combined treatment being more optimal than isolated treatments [43]. A search in PROSPERO, The Cochrane Library and in PubMed was performed and identified no systematic reviews comparing the effectiveness of combined ESWT and any type of exercise including stretching or resistance training versus other common treatment methods for PHP. Therefore, the aim of this work is to conduct such a systematic review and meta-analysis to synthesise the available evidence and inform clinical recommendations regarding the combination of ESWT and exercise interventions for treating PHP. This systematic review and meta-analysis will be conducted in accordance with the Joanna Briggs Institute (JBI) methodology for systematic reviews of effectiveness and will be conducted in accordance with an a priori protocol, which has been registered in the PROSPERO database (CRD42020213286) [44].

\section{Review question}

What is the effectiveness of combined extracorporeal shockwave therapy (ESWT) and exercise interventions compared with other treatments in treating plantar heel pain? 


\section{Inclusion Criteria}

\section{Participants}

This review will consider studies with adult participants aged 18 years old or over, formally diagnosed with PHP. Studies using local anaesthesia will be excluded as research has demonstrated that it can diminish ESWT effectiveness [28]. Studies in which participants have the following ESWT contraindications will also be excluded: diabetes mellitus, systematic inflammatory disease, previous foot surgery or fractures, malignancy, neurological disorders or pregnancy [45].

\section{Interventions}

This review will include studies that have investigated the effectiveness of a combined intervention of any type of ESWT and any type of exercise in the treatment of PHP. Exercise can include cardiovascular exercise, stretching, strengthening, heavy slow resistance training, plyometric, or combined exercise interventions. Any healthcare setting including physiotherapy or podiatry clinics and departments, outpatient departments, primary care settings, specialist orthopaedic or surgical clinics, and rehabilitation clinics will be permitted.

\section{Comparators}

The following comprehensive list of common treatment methods will be included as comparators to combined ESWT and exercise:

1. Self-management, education and advice

2. Corticosteroid injection or topical corticosteroid

3. Stem-cell injection therapies

4. Blood derived injection therapies - platelet-rich-plasma or autologous conditioned plasma

5. Radial or focused ESWT (not combined with exercise)

6. Exercise intervention: including any type of single exercise (cardiovascular, stretching, strengthening, plyometric, heavy slow resistance training) or combined exercise approach (not combined with ESWT) 
7. Standard/usual care or physiotherapy

8. Ultrasound

9. Low level laser therapy (LLLT) or photo-biomodulation therapy

10. Dry needling

11. Acupuncture

12. Manual/manipulative therapy or massage

13. Custom or standard orthotics, insoles or heel cups

14. Botulinum toxin type $A$ injection

15. Taping, bracing, or splinting

16. Surgery, including endoscopic plantar fasciotomy, open plantar fascia release or radiofrequency ablation

17. Combinations of two or more of treatments 1-16

18. Combined ESWT and exercise further combined with one or more of treatments 1-16.

Trials with two or more arms will be included. The use of active co-interventions such as pain medication (NSAIDs), education, advice, orthotics, insoles and any type of exercise or physical activity will be accepted if used in all arms of the trial.

\section{Outcomes}

Primary outcomes will include heel pain and foot function. Heel pain evaluated by any validated scale such as the Visual Analogue Scale (VAS), Numeric Rating Scale (NRS), verbal rating scales or Foot Function Index pain subscale (FFI-PS) will be included $[46,47]$. Foot function evaluated by any validated scale for PHP such as the Foot Function Index (FFI) or Foot and Ankle Ability Measure (FAAM) will be included [48, 49]. Additional secondary outcomes that will be considered for inclusion if available include quality of life measures using validated scales such as the EQ-5D-5L [50].

\section{Types of studies}

The review will be restricted to randomized controlled trials (RCTs) in which combined ESWT and exercise formed one arm of the trial. Trials with two or more 
arms will be considered for inclusion. The use of active co-interventions such as pain medication (NSAIDs), education, orthotics and exercise will be acceptable if used in all trial arms, to limit confounding [51]. In the hierarchy of evidence, systematic reviews of RCTs offer the highest level of evidence [52]. The strongest inferences can be drawn if the review is well conducted and includes methodologically sound RCTs with consistent results [53]. The authors preliminary work has identified several potentially eligible RCTs. Therefore, due to the availability of RCTs on this topic, they will be chosen for inclusion over less robust study designs. Any deviation from the standard RCT design such as crossover or cluster designed trials will also be included.

\section{Methods}

The systematic review process will follow the Preferred Reporting Items for Systematic Reviews and Meta-Analyses (PRISMA) and JBI systematic reviews of effectiveness guidelines [44]. The review has been registered in the PROSPERO database (CRD42020213286).

\section{Search strategy}

The search strategy will seek to identify published and unpublished trials utilizing a three-step search strategy. An initial scoping search of Medline will be conducted (Appendix 1), followed by analysis of text words contained in the title and abstract and article index terms. A comprehensive systematic search using all identified keywords and index terms will then be conducted using the following databases: Medline, CINAHL, AMED, SPORTDiscus, PEDro, Cochrane CENTRAL. The search for unpublished studies will include EThOS Networked Digital Library of Theses and Dissertations and the NICE Guidelines ESWT recommendations. The trial registers to be searched include: ClinicalTrials.gov, UK clinical trials gateway, EU trials registry. Finally, in addition to the comprehensive search, supplementary searches will be undertaken from reviewing bibliographies of articles selected for critical appraisal and related systematic reviews to find those not initially identified. The search strategy will be adapted to each database and be limited to the year 2000 
onwards. The year 2000 was chosen to ensure seminal work was not missed as research on ESWT for PHP first began around this time. Studies published in a language other than English will only be considered if a translation is available as translation services are not available to the authors.

\section{Study selection}

All identified citations from the systematic search will be uploaded into RefWorks (Proquest LLC), with duplicates removed. Two reviewers will independently screen the titles and abstracts of all studies obtained against the identified inclusion criteria. Full-text versions of eligible studies will be accessed and reviewed against the inclusion criteria. Studies will be removed from the screening process if the information provided does not meet the criteria. The details of studies meeting the criteria will be imported in to Covidence [44].

\section{Assessment of methodological quality}

Included studies will be critically appraised by two independent reviewers at study level for methodological quality in the review using the standardized Cochrane risk of bias tool on Covidence [54]. Any disagreements that arise between the reviewers will be resolved through discussion or with a third reviewer. The results of critical appraisal will be reported in narrative form, and in a table. The critical appraisal results will be presented in a table and narrative form. All studies meeting inclusion criteria, regardless of their methodological quality, will undergo data extraction and synthesis and be included in the review.

\section{Data extraction}

Data will be extracted from papers included in the review using the standardised data extraction tool available on Covidence by two independent reviewers [44]. The data extracted will include specific details relative to the interventions, comparators, populations, study methods and outcomes of significance to the review question which include heel pain and foot function. Any disagreements that arise between the reviewers will be resolved through discussion or with a third 
reviewer. Authors of papers will be contacted to request missing or additional data, where required.

\section{Data synthesis}

Quantitative data will, where possible be pooled in statistical meta-analysis using RevMan software. All results will be subject to double data entry. Effect sizes expressed as odds ratio (for categorical data) and weighted mean differences (for continuous data) and their $95 \%$ confidence intervals will be calculated for analysis. Heterogeneity will be assessed statistically using the standard $X^{2}$ and explored using subgroup analyses if possible based on the different quantitative study designs included in this review. Where statistical pooling is not possible due to heterogeneity, the findings will be presented in narrative form including tables and figures to aid in data presentation where appropriate. Analysis of subgroups or subsets is not planned, although the sources of any heterogeneity detected will be explored using subgroup analyses based on the different quantitative study designs included in the review.

\section{Assessing certainty in the findings}

A 'summary of findings' table will be created following The Grading of Recommendations, Assessment, Development and Evaluation (GRADE) approach for assessing the quality of evidence [55]. Evidence from RCTs starts at high quality and the certainty is increased or decreased for several reasons, such as risk of bias [56]. The outcomes reported in the summary of findings table will include heel pain and foot function for the interventions. For each outcome, a ranking of 'high', 'moderate', 'low' or 'very low' will be assigned to the quality of evidence based on the risk of bias. There is by necessity a considerable amount of subjectivity in each decision as GRADE cannot be implemented mechanically. However, GRADE does provide a reproducible and transparent framework for grading certainty in evidence [57]. 


\section{Acknowledgments}

None.

Funding: No sources of funding were used to assist in the preparation of this article.

Conflicts of interest/Competing interests: The author declares no conflicts of interest relevant to the content of this review.

Authorship contributions: IB conceptualised the work, wrote the first draft of the manuscript, revised the manuscript and approved the final manuscript. 


\section{References}

1. Safiri S, Kolahi AA, Cross M, Carson-Chahhoud K, Almasi-Hashiani A, Kaufman J, et al. Global, regional, and national burden of other musculoskeletal disorders 1990-2017: Results from the global burden of disease study 2017. Rheumatology (Oxford). 2020 Aug 25.

2. Sebbag E, Felten R, Sagez F, Sibilia J, Devilliers H, Arnaud L. The world-wide burden of musculoskeletal diseases: A systematic analysis of the world health organization burden of diseases database. Ann Rheum Dis. 2019 Jun; 78(6):844-8.

3. Hoy DG, Smith E, Cross M, Sanchez-Riera L, Blyth FM, Buchbinder R, et al. Reflecting on the global burden of musculoskeletal conditions: Lessons learnt from the global burden of disease 2010 study and the next steps forward. Ann Rheum Dis. 2015 Jan;74(1):4-7.

4. March L, Smith EU, Hoy DG, Cross MJ, Sanchez-Riera L, Blyth F, et al. Burden of disability due to musculoskeletal (MSK) disorders. Best Pract Res Clin Rheumatol. 2014 Jun;28(3):353-66.

5. Blyth FM, Briggs AM, Schneider CH, Hoy DG, March LM. The global burden of musculoskeletal pain-where to from here? Am J Public Health. 2019 Jan;109(1):35-40.

6. Trojian T, Tucker AK. Plantar fasciitis. Am Fam Physician. 2019 06/15;99(12):744-50.

7. Karagounis P, Tsironi M, Prionas G, Tsiganos G, Baltopoulos P. Treatment of plantar fasciitis in recreational athletes: Two different therapeutic protocols. Foot Ankle Spec. 2011 Aug;4(4):226-34.

8. Al-Boloushi $Z$, López-Royo MP, Arian M, Gómez-Trullén EM, Herrero P. Minimally invasive non-surgical management of plantar fasciitis: A systematic review. Journal of Bodywork \& Movement Therapies. 2019 01;23(1):122-37. 
9. Hasegawa M, Urits I, Orhurhu V, Orhurhu MS, Brinkman J, Giacomazzi S, et al. Current concepts of minimally invasive treatment options for plantar fasciitis: A comprehensive review. Curr Pain Headache Rep. 2020 Aug $12 ; 24(9): 55,020-00883-7$.

10. Hansen L, Krogh TP, Ellingsen T, Bolvig L, Fredberg U. Long-Term Prognosis of Plantar Fasciitis: A 5- to 15-Year Follow-up Study of 174 Patients With Ultrasound Examination. Orthop J Sports Med 2018 Mar $6 ; 6(3): 2325967118757983$.

11. Gollwitzer H, Saxena A, DiDomenico LA, Galli L, Bouche RT, Caminear DS, et al. Clinically relevant effectiveness of focused extracorporeal shock wave therapy in the treatment of chronic plantar fasciitis: A randomized, controlled multicenter study. J Bone Joint Surg Am. 2015 May 6;97(9):701-8.

12. Monteagudo M, de Albornoz PM, Gutierrez B, Tabuenca J, Alvarez I. Plantar fasciopathy: A current concepts review. EFORT Open Rev. 2018 Aug $29 ; 3(8): 485-93$.

13. Riel H, Jensen MB, Olesen JL, Vicenzino B, Rathleff MS. Self-dosed and predetermined progressive heavy-slow resistance training have similar effects in people with plantar fasciopathy: A randomised trial. J Physiother. 2019 Jul;65(3):144-51.

14. Rasenberg N, Bierma-Zeinstra SMA, Fuit L, Rathleff MS, Dieker A, van Veldhoven $P$, et al. Custom insoles versus sham and GP-led usual care in patients with plantar heel pain: Results of the STAP-study - a randomised controlled trial. Br J Sports Med. 2020 Sep 2.

15. Salvioli S, Guidi M, Marcotulli G. The effectiveness of conservative, nonpharmacological treatment, of plantar heel pain: A systematic review with meta-analysis. Foot (Edinb). 2017 Dec; 33:57-67. 
16. Shetty SH, Dhond A, Arora M, Deore S. Platelet-rich plasma has better long-term results than corticosteroids or placebo for chronic plantar fasciitis: Randomized control trial. J Foot Ankle Surg. 2019 Jan;58(1):42-6.

17. Jastifer JR, Catena F, Doty JF, Stevens F, Coughlin MJ. Low-level laser therapy for the treatment of chronic plantar fasciitis: A prospective study. Foot Ankle Int. 2014 Jun;35(6):566-71.

18. Sun $\mathrm{K}$, Zhou H, Jiang W. Extracorporeal shock wave therapy versus other therapeutic methods for chronic plantar fasciitis. Foot Ankle Surg. 2020 Jan;26(1):33-8.

19. Babatunde OO, Legha A, Littlewood C, Chesterton LS, Thomas MJ, Menz $H B$, et al. Comparative effectiveness of treatment options for plantar heel pain: A systematic review with network meta-analysis. $\mathrm{Br}$ J Sports Med. 2019 $02 ; 53(3): 182-94$.

20. Digiovanni BF, Nawoczenski DA, Malay DP, Graci PA, Williams TT, Wilding $\mathrm{GE}$, et al. Plantar fascia-specific stretching exercise improves outcomes in patients with chronic plantar fasciitis. A prospective clinical trial with two-year follow-up. J Bone Joint Surg Am. 2006 Aug;88(8):1775-81.

21. Rathleff MS, Molgaard CM, Fredberg U, Kaalund S, Andersen KB, Jensen $\Pi$, et al. High-load strength training improves outcome in patients with plantar fasciitis: A randomized controlled trial with 12-month follow-up. Scand J Med Sci Sports. 2015 Jun;25(3):e292-300.

22. Riel H, Vicenzino B, Jensen MB, Olesen JL, Holden S, Rathleff MS. The effect of isometric exercise on pain in individuals with plantar fasciopathy: $A$ randomized crossover trial. Scand J Med Sci Sports. 2018 Sep 11.

23. Huffer D, Hing W, Newton R, Clair M. Strength training for plantar fasciitis and the intrinsic foot musculature: A systematic review. Phys Ther Sport. 2017 Mar;24:44-52. 
24. Kamonseki DH, Goncalves GA, Yi LC, Junior IL. Effect of stretching with and without muscle strengthening exercises for the foot and hip in patients with plantar fasciitis: A randomized controlled single-blind clinical trial. Man Ther. 2016 Jun;23:76-82.

25. Mani-Babu S, Morrissey D, Waugh C, Screen H, Barton C. The effectiveness of extracorporeal shock wave therapy in lower limb tendinopathy: A systematic review. Am J Sports Med. 2015 Mar;43(3):752-61.

26. Romeo P, Lavanga V, Pagani D, Sansone V. Extracorporeal shock wave therapy in musculoskeletal disorders: A review. Med Princ Pract. 2014;23(1):7-13.

27. Gerdesmeyer L, Frey C, Vester J, Maier M, Weil L,Jr, Weil LS, et al. Radial extracorporeal shock wave therapy is safe and effective in the treatment of chronic recalcitrant plantar fasciitis: Results of a confirmatory randomized placebo-controlled multicenter study. Am J Sports Med. 2008 Nov;36(11):2100-9.

28. Lou J, Wang S, Liu S, Xing G. Effectiveness of extracorporeal shock wave therapy without local anesthesia in patients with recalcitrant plantar fasciitis: A meta-analysis of randomized controlled trials. Am J Phys Med Rehabil. 2017 Aug;96(8):529-34.

29. Li H, LV H, Lin T. Comparison of efficacy of eight treatments for plantar fasciitis: A network meta-analysis. J Cell Physiol. 2018 01;234(1):860-70.

30. Li H, Xiong Y, Zhou W, Liu Y, Liu J, Xue H, et al. Shock-wave therapy improved outcome with plantar fasciitis: A meta-analysis of randomized controlled trials. Arch Orthop Trauma Surg. 2019 12;139(12):1763-70.

31. Li X, Zhang L, Gu S, Sun J, Qin Z, Yue J, et al. Comparative effectiveness of extracorporeal shock wave, ultrasound, low-level laser therapy, noninvasive interactive neurostimulation, and pulsed radiofrequency treatment for treating plantar fasciitis: A systematic review and network meta-analysis. Medicine (Baltimore). 2018 10;97(43):e12819-. 
32. Xiong Y, Wu Q, Mi B, Zhou W, Liu Y, Liu J, et al. Comparison of efficacy of shock-wave therapy versus corticosteroids in plantar fasciitis: A meta-analysis of randomized controlled trials. Arch Orthop Trauma Surg. 2019 $04 ; 139(4): 529-36$.

33. Rompe JD, Furia J, Cacchio A, Schmitz C, Maffulli N. Radial shock wave treatment alone is less efficient than radial shock wave treatment combined with tissue-specific plantar fascia-stretching in patients with chronic plantar heel pain. Int J Surg. 2015 Dec;24(Pt B):135-42.

34. van der Worp $H$, van den Akker-Scheek I, van Schie H, Zwerver J. ESWT for tendinopathy: Technology and clinical implications. Knee Surg Sports Traumatol Arthrosc. 2013 Jun;21(6):1451-8.

35. Ueberle F, Rad AJ. Characterization of unfocused / weakly focused pressure pulse sources for extracorporeal pain therapy ("radial shock wave therapy" sources). Biomed Tech (Berl). 2013 Sep 7.

36. Moya D, Ramon S, Schaden W, Wang CJ, Guiloff L, Cheng JH. The role of extracorporeal shockwave treatment in musculoskeletal disorders. J Bone Joint Surg Am. 2018 Feb 7;100(3):251-63.

37. Wang CJ, Cheng JH, Chou WY, Hsu SL, Chen JH, Huang CY. Changes of articular cartilage and subchondral bone after extracorporeal shockwave therapy in osteoarthritis of the knee. Int J Med Sci. 2017 Feb 23;14(3):21323.

38. Lohrer H, Nauck T, Korakakis V, Malliaropoulos N. Historical ESWT paradigms are overcome: A narrative review. Biomed Res Int. $2016 ; 2016: 3850461$.

39. Gaida JE, Cook J. Treatment options for patellar tendinopathy: Critical review. Curr Sports Med Rep. 2011 Sep-Oct;10(5):255-70. 
40. Takla MKN, Rezk SSR. Clinical effectiveness of multi-wavelength photobiomodulation therapy as an adjunct to extracorporeal shock wave therapy in the management of plantar fasciitis: a randomized controlled trial. Lasers Med Sci 2019;34:583-93.

41. Johannsen FE, Herzog RB, Malmgaard-Clausen NM, Hoegberget-Kalisz M, Magnusson SP, Kjaer M. Corticosteroid injection is the best treatment in plantar fasciitis if combined with controlled training. Knee Surg Sports Traumatol Arthrosc. 2019 Jan;27(1):5-12.

42. Cinar E, Saxena S, Uygur F. Combination therapy versus exercise and orthotic support in the management of pain in plantar fasciitis: A randomized controlled trial. Foot Ankle Int. 2018 Jan 1:1071100717747590.

43. Cinar E, Saxena S, Akkurt HE, Uygur F. Extracorporeal shockwave therapy in the management of plantar fasciitis: A randomized controlled trial. Foot (Edinb). 2020 Sep;44:101679.

44. Aromataris E, Munn Z. e. Joanna Briggs Institute reviewer's manual [Internet]. The Joanna Briggs Institute, The University of Adelaide South Australia; 2020 [cited 2020 Oct 29]: [487 p.]. Available from:

https://reviewersmanual.joannabriggs.org/

45. Ibrahim MI, Donatelli RA, Hellman M, Hussein AZ, Furia JP, Schmitz C. Long-term results of radial extracorporeal shock wave treatment for chronic plantar fasciopathy: A prospective, randomized, placebo-controlled trial with two years follow-up. J Orthop Res. 2017 07;35(7):1532-8.

46. Budiman-Mak E, Conrad KJ, Mazza J, Stuck RM. A review of the foot function index and the foot function index - revised. J Foot Ankle Res. 2013 Feb $1 ; 6(1): 5,1146-6-5$.

47. Boonstra AM, Schiphorst Preuper HR, Reneman MF, Posthumus JB, Stewart RE. Reliability and validity of the visual analogue scale for disability in patients with chronic musculoskeletal pain. Int J Rehabil Res. 2008 Jun;31(2):165-9. 
48. Budiman-Mak E, Conrad K, Stuck R, Matters M. Theoretical model and rasch analysis to develop a revised foot function index. Foot Ankle Int. 2006 Jul;27(7):519-27.

49. Martin RL, Davenport TE, Reischl SF, McPoil TG, Matheson JW, Wukich DK, et al. Heel pain-plantar fasciitis: Revision 2014. J Orthop Sports Phys Ther. 2014 Nov;44(11):A1-33.

50. Herdman M, Gudex C, Lloyd A, Janssen M, Kind P, Parkin D, et al. Development and preliminary testing of the new five-level version of EQ-5D (EQ-5D-5L). Qual Life Res. 2011 Dec;20(10):1727-36.

51, Arah OA. Bias analysis for uncontrolled confounding in the health sciences. Annu Rev Public Health. 2017 Mar 20;38:23-38.

52. Guyatt G, Oxman AD, Akl EA, Kunz R, Vist G, Brozek J, et al. GRADE guidelines: 1 . introduction-GRADE evidence profiles and summary of findings tables. J Clin Epidemiol. 2011 Apr;64(4):383-94.

53, Charrois TL. Systematic reviews: What do you need to know to get started? Can J Hosp Pharm. 2015 Mar-Apr;68(2):144-8.

54. Roque M, Martinez-Garcia L, Sola I, Alonso-Coello P, Bonfill X, Zamora J. Toolkit of methodological resources to conduct systematic reviews. F1000Res. 2020 Feb 4;9:82.

55. Balshem $H$, Helfand $M$, Schunemann HJ, Oxman AD, Kunz R, Brozek J, et al. GRADE guidelines: 3. rating the quality of evidence. J Clin Epidemiol. 2011 Apr;64(4):401-6.

56. Guyatt GH, Oxman AD, Schunemann HJ, Tugwell P, Knottnerus A. GRADE guidelines: A new series of articles in the journal of clinical epidemiology. J Clin Epidemiol. 2011 Apr;64(4):380-2. 
57. Mustafa RA, Santesso N, Brozek J, Akl EA, Walter SD, Norman G, et al. The GRADE approach is reproducible in assessing the quality of evidence of quantitative evidence syntheses. J Clin Epidemiol. 2013 Jul;66(7):736,42; quiz 742.e1-5. 


\section{Appendices}

\section{Appendix 1: MEDLINE search strategy:}

Full search strategy for Medline (EBSCOhost). Search conducted on $16^{\text {th }}$ Aug 2020.

\begin{tabular}{|l|l|l|}
\hline Search & Query & $\begin{array}{l}\text { Records } \\
\text { retrieved }\end{array}$ \\
\hline$\# 1$ & $\begin{array}{l}\text { MH extracorporeal shockwave therapy OR KW } \\
\text { shock wave therapy OR KW shockwave therapy OR } \\
\text { KW shock wave treatment OR KW shockwave* OR } \\
\text { KW ESWT }\end{array}$ & 4,025 \\
\hline \#2 & $\begin{array}{l}\text { MH fasciitis, plantar OR KW plantar OR KW heel } \\
\text { pain OR KW plantar fasciitis OR KW plantar } \\
\text { fasciopathy }\end{array}$ & 14,669 \\
\hline MH & $\begin{array}{l}\text { MH exercise OR MH physical therapy modalities OR } \\
\text { physiotherapy OR KW physical therapy OR KW } \\
\text { usual care OR KW standard care OR KW stretching } \\
\text { OR KW strengthening OR KW corticosteroid }\end{array}$ & 350,869 \\
\hline$\# 4$ & $\begin{array}{l}\text { \#1 AND \#2 AND \#3 } \\
\text { Limited to English language, year 2000 - 2020 }\end{array}$ & 112 \\
\hline
\end{tabular}

